\title{
Resolvendo uma equação de múltiplos pontos utilizando métodos de otimização
}

\author{
Emerson Vitor Castelani \\ Depto de Matemática, UEM \\ 87020-900, Maringá, PR \\ E-mail: emersonvitor@gmail.com
}

\section{André L. M. Martinez}

Depto de Matemática, UTFPR

86300-000, Cornélio Procópio, PR

E-mail: andrelmmartinez@yahoo.com.br

\begin{abstract}
Resumo: No presente trabalho consideramos um estudo numérico sobre uma equação diferencial de segunda ordem com múltiplos pontos de fronteira. Classicamente, este problema é resolvido por métodos numéricos do tipo ponto fixo. Contudo, neste caso, aspectos qualitativos da solução podem não ser considerados. Neste sentido, apresentamos uma nova estratégia baseada em modelos de programação não linear que permitem resolver a equação com múltiplos pontos e, também, impor restrições que exploram os aspectos qualitativos da solução.
\end{abstract}

Palavras-chave: equações diferenciais, múltiplos pontos, programação não linear

\section{Introdução}

Neste artigo investigamos alguns aspectos numéricos relacionados à equação de segunda ordem com múltiplos de fronteira dada por:

$$
\begin{gathered}
u^{\prime \prime}=f\left(t, u, u^{\prime}\right) \\
u(0)=0, u(1)=g\left(u\left(\eta_{1}\right), \ldots, u\left(\eta_{n}\right)\right)
\end{gathered}
$$

onde $f: \mathbb{R}^{3} \rightarrow \mathbb{R}, g: \mathbb{R}^{n} \rightarrow \mathbb{R}$ são contínuas e, possivelmente, não lineares.

Importantes aplicações estão relacionadas com esta equação. De acordo com Lin e Cui [9], variações deste problema surgem no contexto de modelos de fluxo elásticos e viscoelásticos e, em Zou et al [15], aplicações relacionadas com designe de pontes são apresentadas.

Devido às diversas aplicações que cercam esta equação, muitos trabalhos são apresentados na literatura em diversas direções. Recomendamos os trabalhos de Il'in e Moiseev [6,7] pois são os trabalhos pioneiros de estudo de existência de soluções para equações deste tipo. Para outros resultados de existência de solução usando técnicas diversas e variações sobre as condições de fronteira e linearidade das funções envolvidas, recomendamos $[4,5,10,14]$.

Com o crescimento dos trabalhos de existência de solução, diversos trabalhos surgiram para obter soluções numéricas para (1). Neste sentido, recomendamos os trabalhos de [3, 9, 15, 11, 12]. Contudo é importante observar que tais trabalhos não caminharam exatamente na direção dos resultados teóricos.

Essencialmente, a maioria dos trabalhos numéricos estão relacionados com o Teorema de Ponto fixo de Banach [1], isto é, a solução numérica obtida é a definida pela sequência iterativa dada pelo teorema citado. Por outro lado, os resultados de existência (incluindo multiplicidade de solução) utilizam outros teoremas de ponto de fixo (por exemplo, Krasnoselskii [8]) que 
fornecem soluções caracterizadas por positividade, concavidade e com estimativas de normas que limitam o tamanho da solução no espaço que está sendo utilizando.

Neste contexto é natural pensarmos em propostas cujo objetivo é encontrar soluções numéricas que respeitem certas restrições de forma que esta solução encontrada se aproxime de soluções garantidas pela teoria.

Assim, apresentamos uma nova estratégia de resolução que transforma a equação discretizada associada a (1) num problema de programação não-linear (PNL). Uma vez formulado o (PNL), utilizamos uma implementação do método de Lagrangiano Aumentado [2] para resolver o novo problema.

Resolver equações diferenciais por métodos de otimização não é novo [13], contudo, normalmente, tais resoluções dependem de uma aproximação para a solução. Em nossa abordagem, não fazemos uso deste tipo de aproximação, pois a estrutura da equação diferencial nos permite representar uma função que, num certo sentido, mede o quanto a solução encontrada está próxima de satisfazer a equação discretizada. Os autores desconhecem uma abordagem para resolver o problema apresentado neste sentido.

O trabalho está organizado da seguinte forma. Na seção 2, apresentamos os aspectos gerais dos métodos mais comuns utilizados para resolver (1) e um resultado teórico que justifica a busca por soluções numéricas mais refinadas, isto é, que cumpram condições não contempladas por resultados associados ao uso do teorema de ponto fixo de Banach. Na seção 3, apresentamos o ponto principal deste trabalho que é a modelagem da equação discretizada num problema de otimização não linear. Na seção 4 apresentamos alguns exemplos numéricos para elucidar o processo definido na seção 3 e por fim, na seção 5, expomos algumas observações finais e perspectivas.

\section{Métodos clássicos}

Iniciamos esta seção observando que as soluções do problema (1) são pontos fixos do operador definido por:

$$
(T u)(x)=\int_{0}^{1} G(x, t) f\left(t, u(t), u^{\prime}(t)\right) d t+g\left(u\left(\eta_{1}\right), \ldots, u\left(\eta_{m-2}\right)\right) x
$$

onde $G$ é a função de Green dada por

$$
G(x, t)=\left\{\begin{array}{ll}
t(1-x), & t \leq x \\
x(1-t), & t \geq x
\end{array} .\right.
$$

Como comentado anteriormente, diversos métodos numéricos para encontrar a solução de (1) são baseados na argumentação acima e amparados por resultados de existência utilizando o teorema de ponto fixo de Banach. A estrutura básica de um algoritmo para resolver a equação é dada a seguir:

\section{Algoritmo 1:}

1. Discretizar a equação utilizando uma malha uniforme $\left\{x_{j}\right\} \in[0,1]$;

2. Escolher uma aproximação inicial $u_{j}^{0}=u^{0}\left(x_{j}\right)$;

3. Para $k=1,2,3, \ldots$

(a) Aproximar $u^{k}\left(\eta_{1}\right), \ldots, u^{k}\left(\eta_{m-2}\right)$ usando interpolação polinomial (spline linear ou cúbico);

(b) Calcular $u_{j}^{\prime k}$ por diferenças finitas centrais;

(c) Calcular $u_{j}^{k+1}=T\left(u_{j}^{k}\right)$ utilizando alguma regra de integração numérica;

4. Teste a convergência. 
O Algoritmo 1 representa uma estratégia que permite encontrar soluções para o problema em questão, porém por ser baseado no teorema de ponto fixo de Banach, não explora nenhum aspecto qualitativo da solução como, por exemplo, concavidade, positividade e limitação de norma. Além disso, a solução encontrada por este algoritmo é bastante limitada pois normalmente encontra-se numa região onde ocorre a contração do operador dado em (2).

Exemplos e resultados encontrados em diversos artigos relatam resultados de existência de solução que exploram alguns aspectos não contemplados pelo teorema de Banach. Normalmente, estas técnicas se utilizam do teorema de Krasnoselskii, que permite encontrarmos solução em cones definidos em espaços de Banach. Para esclarecer nossa argumentação, considere o seguinte resultado encontrado em [11], cuja demonstração, embora omitida neste texto, utiliza o teorema de Krasnoselskii num cone específico.

Para este resultado, devemos assumir algumas hipóteses básicas que são fornecidas a seguir:

(H1) Existem constantes positivas $\alpha, A, B$ tais que:

$$
\begin{aligned}
& \max _{(t, u, v) \in[0,1] \times[-\alpha, \alpha] \times[-\alpha, \alpha]}\{|f(t, u, v)|\}<\frac{\alpha A}{d_{1}}, \\
& \text { onde } d_{1}=\max _{x \in[0,1]}\left\{\int_{0}^{1}\left|\partial_{x} G(x, t)\right| d t\right\} \\
& \text { - }|g(y)| \leq \alpha B, \forall y \in[0, \alpha]^{m-2} ; \\
& \text { - } A+B \leq 1 .
\end{aligned}
$$

(H2) $f(t, u, v) \geq 0, \forall(t, u, v) \in[0,1] \times[0, \alpha] \times[-\alpha, \alpha]$;

(H3) $g(y) \geq 0, \forall y \in[0, \alpha]^{m-2}$;

(H4) Existe uma constante $\beta$, tal que, $0<\beta<\alpha$ e

$$
\min _{[\bar{m}-1, \bar{m}] \times[0, \beta] \times[-\beta, \beta]} f(t, u, v) \geq \frac{\beta}{\bar{m} d_{2}},
$$

$$
\text { onde } \bar{m} \in\left[0, \frac{1}{2}\right] \text { e } d_{2}=\int_{\bar{m}}^{1-\bar{m}} G(t, t) d t .
$$

Teorema 1. Nas condições definidas por $(H 1)-(H 4)$, o problema (1) tem uma solução $u^{*} \in E$, tal que $\beta \leq\left\|u^{*}\right\|_{E} \leq \alpha$.

O conjunto $E$ apresentado no Teorema 1 é definido como sendo o espaço de Banach das funções continuamente diferenciáveis, ie, $E=C^{1}[0,1]$. A norma que equipa este espaço é dada por:

$$
\|u\|_{E}=\max \left\{\|u\|_{\infty},\left\|u^{\prime}\right\|_{\infty}\right\} .
$$

Ao analisarmos a literatura sobre a equação dada em (1) podemos perceber que, se por um lado resultados de existência de soluções são amplamente explorados, por outro lado, métodos numéricos que permitem encontrarmos soluções caracterizadas por certas especificidades não são usuais. Neste sentido, vamos apresentar na seção seguinte um modelo de programação não linear que nos permite preencher esta lacuna.

\section{Modelagem do problema}

Considere $\left\{x_{j}\right\}, j=0,1 \ldots, n$ uma discretização de $[0,1]$ igualmente espaçada. Denotemos $u_{j}$ as aproximações para $u\left(x_{j}\right)$. Utilizando as fórmulas de diferenças finitas centrais para primeira e segunda ordem queremos, naturalmente, que a solução numérica satisfaça as seguintes identidades:

$$
\frac{u_{j+1}-2 u_{j}+u_{j-1}}{h^{2}}=f\left(x_{j}, u_{j}, \frac{u_{j+1}-u_{j-1}}{2 h}\right), j=1, \ldots, n-1
$$




$$
u_{0}=0,
$$

onde $h=\frac{1}{n}$.

A formulação apresentada em (3)-(4) é um sistema não linear e, classicamente, é utilizada na resolução de equações de segunda ordem. Contudo, considerando a equação (1), a solução não pode ser encontrada dessa forma. O problema reside no fato de que precisamos impor que

$$
u_{n} \approx g\left(u\left(\eta_{1}\right), \ldots, u\left(\eta_{m-2}\right)\right)
$$

e, ao mesmo tempo, calcular aproximações para $u\left(\eta_{1}\right), \ldots, u\left(\eta_{m-2}\right)$. Neste sentido, considere $u_{n+1}, \ldots, u_{n+m-2}$ aproximações para $u\left(\eta_{1}\right), \ldots, u\left(\eta_{m-2}\right)$ a serem determinadas.

Seja $i \in\{1, \ldots, m-2\}$. Sabemos que o valor de $u\left(\eta_{i}\right)$ deve se aproximar de $r\left(\eta_{i}\right)$, onde $r$ é um polinômio interpolador de grau 1. Assim, podemos formular uma função que nos permita fazer uma aproximação para todos os valores $u\left(\eta_{1}\right), \ldots, u\left(\eta_{m-2}\right)$.

Para este propósito considere $\left\{s_{1}, \ldots, s_{m-2}\right\} \subset\{0,1, \ldots, n\}$ tal que

$$
u\left(x_{s_{i}}\right)<u\left(\eta_{i}\right)<u\left(x_{s_{i}+1}\right), i=1, \ldots, m-2,
$$

onde $s_{i}$ é o maior elemento de $\{0, \ldots, n\}$ que satisfaz esta propriedade. Assim, defina

$$
e\left(u_{1}, \ldots, u_{n}, \ldots, u_{n+m-2}\right)=e(u)=\left(r_{1}(u)\right)^{2}+\ldots\left(r_{m-2}(u)\right)^{2},
$$

onde

$$
r_{i}(u)=\left(u_{n+1}-u_{s_{i}+1}-\frac{u_{s_{i}+1}-u_{s_{i}}}{h}\left(\eta_{i}-x\left(s_{i}\right)\right)\right)^{2}, i=1, \ldots m-2 .
$$

Dessa forma, podemos construir o seguinte modelo de programação não linear

$$
\begin{gathered}
\min e(u) \\
\text { s. a. } u_{n}-g\left(u_{n+1}, \ldots, u_{n+m-2}\right)=0 \\
u_{j+1}-2 u_{j}+u_{j-1}-h^{2} f\left(x_{j}, u_{j}, \frac{u_{j+1}-u_{j-1}}{2 h}\right)=0, j=1, \ldots, n-1 \\
u_{0}=0 .
\end{gathered}
$$

Podemos, ainda, impor condições adicionais que nos permitam resolver (1) levando-se em conta outros aspectos da solução. Por exemplo, suponha que a solução que estamos procurando é dada pelo Teorema 1 e que conhecemos alguma estimativa para os valores de $\alpha$ e $\beta$ que limitam a norma da solução. Algumas condições adicionais podem ser impostas no sentido de induzir a solução numérica a satisfazer restrições aproximadas de norma, positividade e concavidade. Por exemplo, podemos impor que

$$
\begin{gathered}
0 \leq u_{i} \leq \alpha, i=1, \ldots, n, \\
0 \leq \frac{u_{i+1}-u_{i-1}}{h} \leq \alpha, i=1, \ldots, n-1 .
\end{gathered}
$$

Naturalmente, o problema definido por (5)-(10) é um problema de otimização com restrições gerais. Uma implementação eficiente que permite resolver este tipo de problema é Algencan ${ }^{1}$. Esta implementação é baseada no método de Lagrangiano Aumentado e permite resolver problemas de programação linear da forma:

$$
\begin{gathered}
\min f(x) \\
\text { s. } a t(x)=0, \\
\\
g(x) \leq 0, \\
a \leq x \leq b,
\end{gathered}
$$

onde $f: \mathbb{R}^{n} \rightarrow \mathbb{R}, h: \mathbb{R}^{n} \rightarrow \mathbb{R}^{m}, g: \mathbb{R}^{n} \rightarrow \mathbb{R}^{p}$ e $a, b \in \mathbb{R}^{n}$. Os passos básicos de métodos do tipo Lagrangiano Aumentado são dados a seguir.

Algoritmo 2: Dado um parâmetro (denominado de penalidade) $\rho>0$, aproximações para $\lambda \in \mathbb{R}^{m}$ e $\mu \in \mathbb{R}_{+}^{p}$ (multiplicadores), faça:

\footnotetext{
${ }^{1}$ Algencan faz parte do projeto tango http://www.ime.usp.br/ egbirgin/tango/
} 
1. Resolva o problema

$$
\begin{gathered}
\min L_{\rho}(x, \lambda, \mu)=f(x)+\frac{\rho}{2}\left\{\sum_{i=1}^{m}\left[h_{i}(x)+\frac{\lambda_{i}}{\rho}\right]^{2}+\sum_{i=1}^{p}\left[\max \left(0, g_{i}(x)+\frac{\mu_{i}}{\rho}\right)\right]^{2}\right\} \\
\text { s.a } a \leq x \leq b .
\end{gathered}
$$

2. Decidir se o ponto obtido no primeiro item pode ser aceito como solução do problema original. Caso o resultado seja afirmativo, parar a execução do algoritmo.

3. De acordo com os resultados dos passos anteriores atualizar os multiplicadores e o parâmetro de penalidade.

4. Voltar ao primeiro passo.

Recomendamos [2] para mais detalhes do método e resultados de convergência.

\section{Exemplos numéricos}

Vamos considerar alguns exemplos numéricos para explorar a capacidade do método. Observe que o objetivo aqui não é fazer uma comparação de velocidade entre um método de ponto fixo e um método de otimização e sim, mostrar como a modelagem apresentada nos permite encontrar soluções numéricas que respeitam algumas características obtidas por resultados teóricos.

No que segue, estaremos entendendo que PTF-Banach representa uma implementação do Algoritmo 1 e Algencan será a implementação usada do Algoritmo 2.

Exemplo 1. Considere a seguinte equação diferencial com cinco pontos de fronteira apresentada de [11].

$$
\begin{gathered}
u^{\prime \prime}=-e^{t}\left(2 t+\frac{3}{10} u+\frac{1}{20} u^{\prime 2}\right) \\
u(0)=0, u(1)=\frac{1}{12}\left(u\left(\eta_{1}\right)+u\left(\eta_{2}\right)+u\left(\eta_{3}\right)\right),
\end{gathered}
$$

onde $\eta=(0.1,0.5,0.8)$.

Neste exemplo, discretizamos e colocamos no formato do modelo de (5)-(8) e portanto, temos um formato implementável em Algencan. Como neste exemplo temos uma estimativa para $\alpha$, podemos complementar o modelo e acrescentar as restrições do tipo (9)-(10). Uma solução numérica para este problema foi obtida em poucas iterações e está representada pela Figura 1.

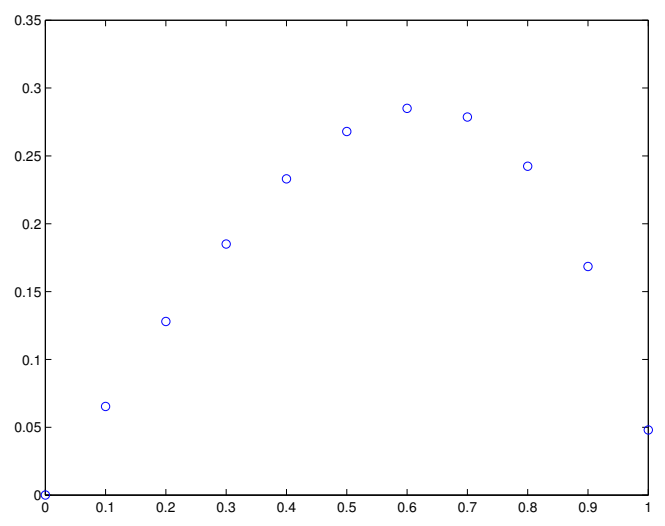

Figura 1: Solução numérica encontrada por Algencan no Exemplo 1. 
Note, pela Figura 1, que o comportamento da solução encontrada respeita as condições fornecidas pelo teorema de existência, isto é, concavidade, positividade e limitação de norma (superior).

Exemplo 2. Vejamos agora um simples exemplo linear com três pontos de fronteira, obtido de $[3]$.

$$
\begin{gathered}
u^{\prime \prime}=-6 x \\
u(0)=0, u(1)=g\left(u_{\eta}\right),
\end{gathered}
$$

onde $g(s)=s / 2$. Neste exemplo, vamos considerar três valores diferentes para $\eta$, a saber, $\eta=0.12, \eta=0.58$ e $\eta=0.98$. As soluções exatas correspondendo aos valores de $\eta$ são respectivamente:

e

$$
\begin{aligned}
& u(t)=\frac{31223}{29375} t-t^{3}, \\
& u(t)=\frac{225611}{177500} t-t^{3} \\
& u(t)=\frac{44117}{42500} t-t^{3} .
\end{aligned}
$$

Para os testes, obtivemos soluções utilizando $h=0.1$, com chute inicial $u^{0}=(0, \ldots, 0)$. A modelagem, segue as mesmas idéias de antes, porém não utilizamos neste exemplo condições de limitação de norma. Os resultados numéricos são expressos na Tabela 1 onde

$$
E_{\text {num }}=\max \left|u^{j}-u\left(t_{j}\right)\right|,
$$

representa o erro absoluto máximo entre a solução numérica do algoritmo utilizado e a solução exata.

\begin{tabular}{ccc}
\hline$\eta$ & $E_{\text {num }}(\mathrm{PTF}-\mathrm{Banach})$ & $E_{\text {num }}($ Algencan $)$ \\
\hline 0.12 & $1.16550 \mathrm{E}-007$ & $3.57489 \mathrm{E}-004$ \\
0.58 & $8.58690 \mathrm{E}-005$ & $1.94174 \mathrm{E}-003$ \\
0.98 & $2.31915 \mathrm{E}-003$ & $4.51899 \mathrm{E}-003$ \\
\hline
\end{tabular}

Tabela 1: Comparação entre os algoritmos com vários valores de $\eta$.

Neste exemplo podemos observar que devido a natureza do problema, PTF-Banach funciona muito bem, obtendo resultados numéricos mais precisos do que Algencan. Contudo, uma observação é pertinente. O PTF-Banach mostra-se muito sensível ao valor de $\eta$, isto é, quanto mais próximo de 1, mais erros iremos acumular durante o processamento do Algoritmo 1 e consequentemente, a última aproximação acumula estes erros. Por outro lado, quando utilizamos Algencan, não ocorre este problema pois, no máximo, o valor da função objetivo será maior mas isso não interfere na uniformidade do método.

\section{Conclusões e trabalhos futuros}

No presente artigo, consideramos uma nova abordagem de resolução numérica para uma equação de segunda ordem com múltiplos pontos de fronteira. A nova modelagem permite obter soluções que contemplam algumas propriedades comuns oriundas de resultados teóricos de existência. Neste sentido, o presente estudo indica algumas possibilidades de se trabalhar com a resolução deste tipo de equação utlizando o método de Lagrangiano Aumentado via implementação Algencan. Os resultados numéricos obtidos até o momento mostraram dois importantes aspectos da nova formulação: a estabilidade quanto as variações nos pontos de fronteira e obtenção de ponto fixo não trivial do operador integral definido em (3). Tais fatos refletem uma abordagem promissora pois aspectos que antes eram explorados apenas no campo teórico passam a ser 
incorporados possibilitando novas perspectivas numéricas de resolução de equações diferenciais ordinárias. Ainda, são objetivos futuros para complementar este trabalho:

- Estabelecer métodos para obtenção de múltiplas soluções;

- Realizar mais testes numéricos para verificar a confiabilidade da nova abordagem;

- Comparar com outros métodos e implementações;

- Expandir a ideia para outras equações.

\section{Referências}

[1] R.P. Agarwal, M. Meehan, D. O'Regan, "Fixed Point Theory and Applications", Cambridge University Press, Cambridge (2001).

[2] R. Andreani, E. G. Birgin, J. M. Martínez and M. L. Schuverdt, On Augmented Lagrangian Methods with general lower-level constraints, SIAM Journal on Optimization 2007.

[3] E.V. Castelani, T.F. Ma, Numerical solutions for a three-point boundary value problem, Communications in Applied Analysis, 11 No. 1 (2007) 87-96.

[4] C.P. Gupta, Solvability of a three-point nonlinear boundary value problem for a second order ordinary differential equation, J. Math. Anal. Appl, 168 No. 2 (1992) 540-551.

[5] C.P. Gupta, S.I. Trofimchuk, A sharper condition for the solvability of a three-point second order boundary value problem, J. Math. Anal. Appl, 205 No. 2 (1997) 586-597.

[6] V.A. Il'in, E.I. Moiseev, Nonlocal boundary-value problem of the secod kind for a SturmLiouville operator, Differential Equations, 23 (1987) 979-987.

[7] V.A. Il'in, E.I. Moiseev, A nonlocal boundary value problem for the Sturm-Liouville operator in the differential and difference treatments, Sov. Math. Dokl, 34 (1987) 507-511.

[8] M. A. Krasnoselskii, Positive solutions of operator equations, Noordhoff, Groningen, 1964.

[9] Y. Lin, M. Cui, A numerical solution to nonlinear multi-point boundary value problems in the reproducing kernel space,Mathematical Methods in the Applied Sciences, 34 (2011) $44-47$.

[10] R. Ma, Existence theorems for a second order $m$-point boundary value problem, J. Math. Anal. Appl, 211 (1997) 545-555.

[11] A. L. M. Martinez, C. A. P. Martinez, E. V. Castelani, T. S. Pinto, Remarks on positive solutions for an m-point boundary value problem, Int. Journal of Appl. Math., 25 (2012) 281-297.

[12] A. L. M. Martinez, C. A. P. Martinez, E. V. Castelani, T. S. Pinto, Um estudo de soluções para um problema de segunda ordem com múltiplos pontos de fronteira. Tendências em Matemática Aplicada e Computacional, 14 (2013) 255-263.

[13] J. M. Martínez, F. Pisnitchenko, S. A. Santos, Métodos de otimização para resolução de equações diferenciais, XXX CNMAC, 2007.

[14] P.J.Y. Wong, R.P. Agarwal, Existence and uniqueness of solutions for three-point boundary value problems for second order difference equations, Proceedings of Dynamic Systems and Applications, Atlanta, United States of America, (1995), 553-560.

[15] Y. Zou, Q. Hu e R. Zhang, On numerical studies of multi-point boundary value problem and its fold bifurcation, App. Math. and Comp., 185 (2007) 527-537. 